\title{
¿Promueven los libros de texto la indagación científica?
}

Do textbooks promote scientific inquiry?

Ana Aragüés ${ }^{1}$

\section{Resumo}

Este trabalho centra-se no estudo de atividades propostas em livros didáticos de ciências de $1^{0}, 3^{\circ}$ e $4^{\circ}$ ano do Ensino Fundamental, correspondendo a três editoras espanholas. Especificamente, é analisado o grau de promoção de estratégias de investigação científica propostas aos alunos. A análise se concentra na categorização da demanda para cada uma das atividades que aparecem na seleção dos livros, de acordo com um sistema de estratégias científicas. Os resultados mostram uma escassa presença de atividades que promovam o conjunto de estratégias, evidenciando uma grande maioria de atividades de preparação para a escrita, descrição ou classificação oral, identificação e união. Consequentemente, há necessidade de mudanças nos livros didáticos condizentes com uma aprendizagem abrangente dos processos que caracterizam a atividade científica.

Palavras chave: livros didáticos; anos iniciais; estratégias; investigação; ciência.

\section{Resumen}

Este trabajo se centra en el estudio de actividades propuestas en los libros de texto de ciencias en $1^{0}, 3^{\circ}$ y $4^{\circ}$ curso de Primaria, correspondientes a tres editoriales de amplia difusión. En concreto, se analiza el grado de promoción de estrategias de indagación científica propuesta a los estudiantes. El análisis se centra en la categorización de la demanda de cada una de las actividades que aparecen en la selección de libros, de acuerdo, de acuerdo a un sistema de estrategias científicos. Los resultados muestran una escasa presencia de actividades que promuevan el conjunto de estrategias, apreciándose una gran mayoría de actividades orientadas hacia el desarrollo de estrategias como son la Elaboración escrita, la Descripción oral o la Clasificación, identificación e unión. En consecuencia, se plantea un cambio en las demandas recogidas en los libros de texto coherente con un aprendizaje integral de los procesos que caracterizan a la ciência.

Palabras clave: libros de texto; primaria; estrategias; indagación; ciência.

\section{Abstract}

This work focuses on the study of the activities proposed in the science textbooks in 1st, 3rd and 4 th grade of Primary, corresponding to three widely distributed publishers. Specifically, the degree of promotion of scientific inquiry strategies proposed to students is analyzed. The analysis focuses on the categorization of the demand for teach of the activities that appear in the selection of books, according to a system of scientific strategies. The results show a scarce presence of activities that promote the set of strategies, appreciating a great

\footnotetext{
${ }^{1}$ Universidad de Zaragoza | aragues.ddcc@gmail.com
} 
majority of activities oriented towards the development of strategies such as written preparation or classification, identification and union. Consequently, there is a change in the demands collected in the textbooks consistent with a comprehensive learning of the processes that characterize science.

Keywords: text-books; primary; inquiry; strategies. Science.

\section{Introduccion}

La sociedad actual y la forma en la que ésta se desarrolla demanda cada vez más una cultura científica ciudadana básica. Los recientes acontecimientos a nivel mundial relacionados con la COVID-19 ponen de manifiesto la necesidad de una alfabetización científica (Acevedo, 2004) que permita a los futuros ciudadanos entender y comprender qué es lo que ocurre a su alrededor. En este sentido, la escuela debe concebirse como una oportunidad para ofrecer a los alumnos la posibilidad de observar el mundo desde un paradigma científico.

En lo que se refiere a enseñanza de las ciencias, son constantes las investigaciones que promueven un enfoque de la enseñanza mediante la indagación en el aula (De la Blanca, Hidalgo y Burgos, 2013; Greca, Meneses Villagrá, y Diez Ojeda, 2017; Postigo Fernández y Greca Dufranc, 2014). Esta consideración es planteada como una forma de aprendizaje activa en donde el alumnado tiene la oportunidad de construir el conocimiento, principalmente a través de la interacción, de la pregunta, así como de los procesos implicados para dar respuesta como son observar, describir o argumentar, entre otros. Resumiendo, se podría decir, que se trata de un aprendizaje que tiene en cuenta no sólo los contenidos científicos sino todo el conjunto de procesos implicados mediante los cuales la ciencia es construida. Además, se suma el hecho que desde es el currículo oficial se insta hacia un aprendizaje del conjunto de los procesos científicos. En este sentido, en la actualidad, las editoriales han aportado nuevos formatos de materiales educativos bajo títulos como Explora, Proyectos, Investiga o Aplica una técnica, tanto en su forma digital como en formato de libro de texto. En otras palabras, las editoriales parecen haber incluido actividades orientadas hacia un trabajo de investigación en el aula.

Desde el rol que el libro de texto parece seguir jugando en el aula (Braga y Belver, 2016), en este trabajo, se analiza el tipo de demanda en relación a los distintos procesos científicos que a través de las actividades se plantean en los libros de texto.

\section{Marco empirico}

\section{La indagación en el aula de Ciencias}

Numerosos estudios reflejan, la importancia de las ciencias como elemento indispensable desde los primeros niveles escolares para la adquisición y comprensión de conocimientos científicos (Rivero, del Pozo y Porlán, 2017). Por otro lado, el currículo actual, explica que la finalidad de las ciencias reside en aportar al alumno conocimientos, actitudes y habilidades para comprender y afrontar mejor la sociedad, alcanzando madurez a la hora de reflexionar y desarrollar sus propios aprendizajes. 
Desde la investigación en didáctica de las ciencias se promueve un aprendizaje basado en cierta medida en cómo la ciencia se construye. En palabras de Gil Pérez (1994) encontramos que uno de los mayores retos de la enseñanza en ciencias es la enorme distancia que existe entre las situaciones de aprendizaje y la forma en que la Ciencia es construida. De esta forma, un aprendizaje de las ciencias debiera pasar también por un aprendizaje de los procesos que la caracterizan. Esta serie de procesos son la observación, la clasificación, la descripción, la argumentación, la predicción, la medición etc. es decir, ese conjunto de tareas que hacen que hacer ciencia pueda definirse como una actividad metódica, rigurosa y basada en la razón.

Tabla 1. Secuencia para desarrollar la comprensión personal: modelo exploración creativa.

\begin{tabular}{ll}
\hline Estrategias de indagación & Acciones implicadas \\
\hline (1) Explorar & Sobre un problema, situación, fenómeno, artefacto, modelo, \\
& acontecimiento, historia... \\
\hline (2) Observar & El fenómeno que se va a tratar de forma global \\
\hline (3) Identificar evidencias & Selección de partes del dispositivo experimental \\
& Aspectos clave que tratar \\
& Cambios que han tenido lugar \\
& Partes implicadas \\
\hline (4) Buscar explicaciones & Qué es lo que hacen estas partes/estructuras \\
& Cuál es la causa y el efecto de los cambios \\
& Cuál es la función \\
& Qué partes están interactuando \\
& Cuál es el resultado de esa interacción \\
& Que tendencias y pautas tienen lugar \\
& Creación de razones personales basadas en la evidencia -en \\
& caso de que haya- \\
\hline Introducción de nuevas variables \\
Planificación de procesos para probarlas: Medir, comparar, \\
verificar, probar, identificar, clarificar. \\
La autoevaluación de estas investigaciones puede conducir a \\
nuevas o modificadas explicaciones, dudas sobre ideas previas \\
o conclusiones provisionales. Estas explicaciones provisionales \\
se comunican a los compañeros para la evaluación de grupo y \\
reestructuración. \\
\hline (5) Evaluar de la explicación \\
situaciones. \\
\hline
\end{tabular}

Fuente: Milne (2008)

No es fácil acotar el número de procesos o tareas que serían esperables en un entorno de aprendizaje de las ciencias por indagación. En este trabajo se hace especial mención al modelo secuenciado de Milne (2008) ya que será un referente para realizar la categorización. El modelo se basa en un aprendizaje de la ciencia basado en los procesos que la caracterizan y definen. Las estrategias de indagación que plantea el modelo son las siguientes: Explora, Observa, Identifica evidencias, Busca explicaciones, Comprueba explicaciones, Evalúa, Más investigaciones (Tabla 1).

La primera estrategia Explorar (1) hace referencia a la toma de contacto inicial que el alumno realiza con el objeto de estudio. Se trata de una situación introductoria que permite al alumno situarse. La estrategia Observar (2) expresa la situación en la que el alumno 
observa el proceso o fenómeno de manera global. A diferencia de la estrategia Explorar (1) implica cierto grado de atención. La estrategia Identifica evidencias (3) la observación de una o más partes del fenómeno. Requiere una mayor atención en cuanto a los aspectos clave que van a ser tratados, cambios producidos, así como la toma de registros o datos en su caso. La siguiente estrategia Buscar explicaciones (4) es aquella en la que los estudiantes establecen relaciones entre partes del sistema de forma causal, funcional, de integración o establecen posibles pautas. La siguiente estrategia es Evaluación de la explicación (5). Esta estrategia aparece cuando las explicaciones son comunicadas al grupo y sometidas a evaluación en la medida de poder ser contrastadas, comparadas o mayormente razonadas en base a lo que se conoce. La estrategia Predice utilizando el conocimiento/ Más investigación (7) se diferencia de lo que supondría la elaboración de hipótesis en la medida que predecir implica el manejo de un marco de conocimiento frente a lo que sería un planteamiento de hipótesis, la cuál puede ser planteada azarosamente. Esta estrategia es identificada cuando los alumnos utilizan una serie de contenidos científicos que han sido trabajados para establecer relaciones que les permitan realizar predicciones sobre una hipotética o futura situación.

\section{El libro de texto como herramienta de aprendizaje}

A lo largo de la historia, el libro de texto ha ido sufriendo modificaciones a medida que las políticas educativas lo han hecho. Su uso en el aula ha supuesto, y todavía supone, una forma de guía tanto para el docente como para el alumno. Estos libros bien podrían considerarse como los portadores del conocimiento que una generación desea transmitir a la siguiente (Fuchs y Bock, 2016). No es de extrañar que se hayan realizado numerosos estudios en este sentido. En concreto, y en lo que compete al área de Didáctica de las Ciencias, destacan aquellos estudios relacionados con las ilustraciones que aparecen en ellos (Palacios y de Dios Jiménez, 2002), los que analizan el papel de las editoriales a la hora de interpretar el currículo oficial (Braga Blanco y Domínguez, 2016), los relacionados con el tratamiento conceptual propuesto (de la Gándara, Gil y Sanmartí, 2001) o aquellos relacionados con el tipo de actividades propuestas para afianzar los conceptos que se pretende que el alumnado adquiera (Martínez Losada y García Barros, 2003). Dentro de este último tipo de estudio, cabe destacar algunos resultados como los que señalan que si bien parecen percibirse mejoras en cuanto al tipo de diseño o de imagen o la naturaleza del tipo de actividades no ha cambiado sustancialmente de forma histórica (Caamaño y Vidal, 2001; Martínez Losada y García Barros, 2003).

\section{Analisis}

El análisis se centra en la categorización de las distintas actividades que aparecen en una selección de libros de texto de Ciencias de Primaria. Para ello, se procedió a la selección de cuatro libros correspondientes a editoriales con una amplia demandada en los centros educativos del contexto español (Santillana, Vicens Vives y Edebé). Se eligieron los niveles de $1^{\circ}, 3^{\circ}$ y $4^{\circ}$ de Primaria, siendo los siguientes:

Ciencias de la Naturaleza $1^{0}$ Primaria Editorial Santillana (2013).

Ciencias Sociales $3^{\circ}$ Primaria Editorial Santillana (2018).

Ciencias Sociales $3^{\circ}$ Primaria Editorial Vicens Vives (2014). 
Ciencias de la Naturaleza $4^{0}$ Primaria Editorial Edebé (2018).

El sistema de categorías empleado fue realizado de forma abierta teniéndose en cuenta el trabajo realizado de Milne (2008) (Tabla 1). Con el fin de obtener una validez en el estudio, tras la elaboración del sistema de categorías se procedió a una triangulación (con una coincidencia mayor del 80\%). A continuación, se presentan las categorías que fueron establecidas:

\section{Observación}

Tiene como objetivo un acercamiento inicial al problema, enfrentando al alumnado al objeto de estudio, generalmente sobre imágenes presentadas en el libro. Ej. Observa el paisaje que se ve desde la ventana de vuestra clase.

\section{Elaboración de dibujo}

Frente a la descripción escrita se encuentra la descripción pictórica. La elaboración de un dibujo permite al alumnado, especialmente en cursos iniciales, concretar procesos observacionales e incluso manifestar detalles que quizá a través de la descripción escrita son pasados por alto. Ej. Observa las manos. Elabora un dibujo de la mano izquierda de tu cuerpo y coloréala.

\section{Clasificación, identificación-unión}

Esta categoría recoge aquellos procesos relacionados con la capacidad de ordenar o de establecer una asociación entre dos cosas distintas, trenzando nexos de unión entre ellas. Ej. Une cada sentido con una parte del cuerpo.

\section{Descripción oral}

Describir de forma oral es explicar cómo es una determinada cosa, persona o lugar. Se trata de una facultad propia del ser humano realizada a diario. Para realizar una buena descripción, primero debemos observar bien una determinada realidad para después analizar lo observado y finalmente poder realizar la descripción de lo que se vio. La descripción oral solicita al alumno una respuesta e incentiva a plasmar lo que han percibido o leído con anterioridad. Ej. Explica a tus compañeros cómo has realizado la tarea planteada.

\section{Elaboración escrita individual}

Hace referencia al desarrollo de una habilidad lingüística relacionada con las capacidades de organización, estructuración y distribución de las ideas. Esta destreza requiere de una serie de subprocesos tales como analizar la situación de comunicación, generar ideas, hacer planes, redactar, revisar y reformular enunciados. Ej. Explica por qué tienen lugar los siguientes procesos.

\section{Elaboración escrita en grupo}

En esta categoría se incluyen aquellas actividades de tipo grupal, en ellas deben cooperar, asociarse, compartir conocimientos o elaborar situaciones trabajando en equipo, 
aumentando su capacidad para alcanzar un objetivo común. Ej. Cuando suena el despertador, ¿cuál es el primer sentido que se activa? Pensadlo y anotarlo.

\section{Argumentación}

En este tipo de categoría se engloban aquellas demandas relacionadas con la elaboración de un razonamiento que es empleado para probar que lo que se dice es cierto. Se pretende que el escolar adquiera la capacidad de sostener un punto de vista fundándolo en la evidencia, en los datos o en la exposición de sus motivos. Ej. Una compañera de tu clase ha sido operada de apendicitis, pero no quiere perderse nada de lo que hagáis en clase ¿cómo podrías ayudarla?, ¿qué otras personas podrían colaborar?, ¿cómo os organizaríais para ello?

\section{Investigación}

La investigación se plantea como una forma de ampliar o desarrollar el conocimiento. Para ello es necesaria la búsqueda y selección de información. Así, se trata de propuestas que hacen referencia a cómo se sabe o cómo se ha llegado a conocer o a hacer una determinada afirmación, y donde se solicita normalmente una comprobación de las hipótesis. Además, se pueden apoyar en las nuevas tecnologías para realizar esta búsqueda de información. Esta investigación puede desarrollarse de manera individual o grupal. Ej. Investiga cómo se llama el fruto de la encina.

\section{Resultados}

En la Tabla 2 se recogen los porcentajes obtenidos de las categorías en cada uno de los libros de texto analizados.

Tabla 2. Representación del porcentaje de categorías relacionadas con la indagación en los libros de texto analizados.

\begin{tabular}{|c|c|c|c|c|c|}
\hline & $\begin{array}{c}\text { Santillana } \\
\text { 10 Primaria } \\
\text { (2013) } \\
\end{array}$ & $\begin{array}{c}\text { Santillana } \\
\text { 3o Primaria } \\
\text { (2018) } \\
\end{array}$ & 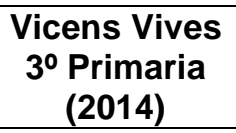 & $\begin{array}{c}\text { Edebé } 4^{\circ} \\
\text { Primaria } \\
(2018)\end{array}$ & Global \\
\hline Observación & 7.8 & 5.7 & 1.0 & 2.5 & 4.3 \\
\hline $\begin{array}{l}\text { Elaboración de un } \\
\text { dibujo }\end{array}$ & - & - & 1.0 & - & 1.0 \\
\hline $\begin{array}{l}\text { Clasificación, } \\
\text { identificación, unión }\end{array}$ & 25.1 & 4.9 & 3.4 & 10.8 & 12.1 \\
\hline Descripción Oral & 19.6 & 18.0 & 9.2 & 4.5 & 11.4 \\
\hline $\begin{array}{l}\text { Elaboración escrita } \\
\text { individual }\end{array}$ & 31.3 & 47.8 & 62.3 & 54.8 & 49.2 \\
\hline $\begin{array}{l}\text { Elaboración escrita en } \\
\text { grupo }\end{array}$ & 7.3 & 10.6 & - & 7.6 & 6.7 \\
\hline Argumentación & 7.3 & 8.2 & 14.0 & 6.4 & 9.1 \\
\hline Investigación & 1.7 & 4.5 & 9.1 & 13.3 & 6.9 \\
\hline
\end{tabular}

Los resultados muestran diferencias significativas entre las estrategias observadas entre $\operatorname{los} 1^{\circ}$ y $4^{\circ}$ de Primaria. Este hecho es atribuido, en parte, al curso escolar para el que cada 
libro está planteado, así como a la madurez de los procesos que supuestamente deben ser adquiridos en cada uno de ellos. Así, parece coherente que en cursos iniciales como $1^{\circ}$ de Primaria, en los que el alumnado está aprendiendo la lectoescritura, exista un mayor número de actividades que demanden una Clasificación, identificación, unión frente a $4^{\circ}$ de Primaria curso en el cual cobra una mayor presencia demandas relacionadas con la Elaboración escrita (Tabla 2). Otras tres categorías con porcentajes elevados han sido la Descripción oral (11,4\%), la Clasificación, identificación, unión (12,1\%) y la Argumentación $(9,1 \%)$ (Tabla 2).

Por otra parte, encontramos que la Observación, la Elaboración de un dibujo, Elaboración escrita en grupo y la Investigación son las categorías con menor presencia en los libros de texto de Primaria. La Observación parece disminuir considerablemente a medida que avanzamos de curso, encontrando diferencias significativas entre la editorial Santillana (5,7\%) y la editorial Vicens Vives (1\%) (Tabla 2). Así, la estrategia de poner la atención en un determinado hecho o fenómeno no parece ser del todo promovida en la selección de libros analizados. Si bien un 7,8\% es asociado a esta categoría en el libro de Santillana $1^{0}$ Primaria (2013), cabe mencionar que se trata de una observación reducida a imágenes presentadas en el libro, distando de ser una observación relacionada con algún fenómeno natural contextualizado. En cuanto a la Elaboración de un dibujo ésta sólo es promovida en el libro de $3^{\circ}$ Primaria Vicens Vives. En principio, sería esperable que la elaboración pictórica fuese promovida principalmente en los primeros cursos debido a la carencia que posee el alumnado en la escritura. Sin embargo, el único libro de texto que promueve actividades basadas en la elaboración de dibujos es el de la editorial Vicens Vives de $3^{\circ}$ de Primaria. Continuando con la categoría relacionada con Elaboración escrita en grupo obtenemos una media de 6,7\%. Este porcentaje se sitúa entre un 7,3 hasta un 10,6 \%, siendo el único libro el de la editorial Vicens Vives en el que no aparece. Respecto a la categoría Elaboración escrita en grupo un porcentaje entre el 7 y el 10\% de las actividades analizadas (a excepción de la editorial Vicens Vives, en cuyo caso la presencia es nula). De ello se deduce que, aunque no en todas editoriales, se aprecia una promoción hacia el trabajo en grupo y, por ende, cierta aproximación a lo que supone la cooperación científica. Por último, se encuentra la Investigación con una media de un 6,9 \%, con un 1,7\% en el libro de Santillana de $1^{\circ}$ Primaria hasta un 13,3\% correspondiente al libro de Santillana $4^{\circ}$ de Primaria (Tabla 2). Esta categoría muestra aumento progresivo a lo largo de los cuatro cursos académicos, aunque cabe indicar que la gran mayoría de las actividades propuestas más que una investigación la demanda va en relación con la búsqueda de información sobre un proceso concreto (ej. Amplía información sobre la diálisis). Por otro lado, se aprecia una notable diferencia den la Investigación entre las editoriales Santillana (18\%) y Vicens Vives $(9,2 \%)$ para $3^{\circ}$ de Primaria.

\section{Conclusiones}

Desde la educación, debemos poner la mirada en nuevos enfoques y metodologías que promuevan, al igual que en ocurre en la ciencia, una indagación que fomente un espíritu científico. En este sentido, el presente trabajo pretende aportar resultados relacionados con las actividades científicas educativas que son planteadas al alumnado, en concreto, las que se recogen en los libros de texto. 
A pesar de las nuevas corrientes metodológicas en lo que concierne al aprendizaje de las ciencias, el libro de texto sigue siendo un vehículo de aprendizaje universal en las aulas (Occelli y Valeiras, 2013). Así, y aunque bien es cierto que el docente posee cierta libertad a la hora de abordar su clase, el libro de texto, y en consecuencia las actividades que este recoge, todavía constituyen una fuente principal del aprendizaje. El análisis mediante categorías del tipo de demanda de las actividades planteadas en este estudio muestra una escasa presencia de actividades que promuevan el conjunto de estrategias científicas. Los resultados muestran cómo principalmente las capacidades que promueven estas actividades se relacionan con la Elaboración escrita, la Descripción oral o la Clasificación, identificación, unión. Procesos como son la Observación o la Investigación están poco presentes en las cuestiones planteadas al alumnado. Además, las escasas actividades relacionadas con la Investigación son en esencia una búsqueda de información a través de herramientas tecnológicas, distando enormemente de lo que supone una investigación científica en donde es pertinente el uso de argumentos o de pruebas.

Podemos concluir que, en general, los libros analizados proponen al estudiante actividades en las que no existe una demanda sobre auténticos planteamientos, sino que más bien se limitan a fomentar una repetición de la información que previamente ha sido presentada. Estos datos coinciden con el estudio realizado por Laya y Martínez (2013) quienes destacan que los libros de texto no inciden suficientemente en habilidades como son la propuesta de estrategias o la elaboración de conclusiones argumentadas. En este sentido, y a la luz de los resultados obtenidos, podemos apreciar cómo aspectos clave para el desarrollo de la indagación en el aula no son del todo promovidos por las actividades propuestas en los libros de texto. Esta situación confirma que pese a los diversos estudios realizados y modelos de nuevas metodologías que existen, las editoriales continúan optando por elaborar actividades cuya demanda se prevé discutible. Por tanto, Desde la Educación Primaria, se hace necesario un cambio en la selección de actividades para una promoción de habilidades relacionadas con el conocimiento científico (Pro Bueno, 2013).

\section{Referencias}

Acevedo, J. A. (2004). Reflexiones sobre las finalidades de la enseñanza de las ciencias: educación científica para la ciudadanía. Revista Eureka sobre Enseñanza y Divulgación de las Ciencias, 1(1), 3-16.

Braga Blanco, G. M. \& Belver Domínguez, J. L. (2016). El análisis de libros de texto: una estrategia metodológica en la formación de los profesionales de la educación. Revista Complutense de Educación, 27(1), 199-218.

Caamaño, A. \& Vidal, F. (2001). Las ciencias de la naturaleza en la ESO. Una visión desde Cataluña. Alambique, 27, 31-43.

De la Blanca, S., Hidalgo, J. y Burgos, C. (2013). Escuela infantil y ciencia: la indagación científica para entender la realidad circundante. Enseñanza de las ciencias, (Extra), 979-983.

De la Gándara, M., Gil, M.J. y Sanmartí, N. (2001). The biological adaptacion model: obstacle or a didactic recourse?, en García Rodeja, I., Díaz Bustamante, J., Harms, U. y Jiménez

Aleixandre, M.P. (eds.). Proceedings of III Conference of European Researchs in Didactic of 
Biology (ERIDOB), pp. 157-168. Santiago de Compostela: Universidad de Santiago de Compostela.

Fuchs, E., \& Bock, A. (Eds.). (2018). The Palgrave handbook of textbook studies. London: Palgrave Macmillan.

García, O. (2014). La didáctica de las ciencias experimentales en Educación Primaria. Una propuesta de intervención. Universidad de Valladolid, España.

Gil Pérez, D. (1994). Relaciones entre conocimiento escolar y conocimiento científico. Revista Investigación en la Escuela, 23, 17-32.

Greca, I. M., Meneses Villagrá, J. A. y Diez Ojeda, M. (2017). La formación en ciencias de los estudiantes del grado en maestro de Educación Primaria. Revista Electrónica de Enseñanza de las Ciencias, 16(2), 231-256.

Laya Iglesias, P., \& Martínez-Losada, C. (2019). La competencia científica en los libros de texto de educación primaria. Ápice. Revista de Educación Científica, 3(1), 71-83.

Martínez Losada, C. y García Barros, S (2003). Las actividades de primaria y ESO, incluidas en libros de texto. ¿Qué objetivos persiguen? ¿Qué procedimientos enseñan? Enseñanza de las Ciencias, 21(2), 243-259.

Milne, I. (2008). 'Creative exploration: doing science in a primary School context'. In imaginative practice, imaginative inquiry, pp. 61-70. Proceedings of the Sixth International Conference on Imagination and Education. Rydges Lakeside, Canberra, Australia, 29-31 January 2008 Edited by Robert Fitzgerald \& Thomas W. Nielsen.

Occeli, M. y Valeiras, N. (2013) Los libros de texto de ciencias como objeto de investigación: una revisión bibliográfica, Enseñanza de las Ciencias, 31(2), pp. 133-152.

Palacios, F. J. P. \& de Dios Jiménez, J. (2002). Las ilustraciones en la enseñanza-aprendizaje de las ciencias. Análisis de libros de texto. Enseñanza de las ciencias, 20(3), 369-386.

Postigo Fernández, D. y Greca Dufranc, I. M. (2014). Uso de la metodología de la indagación para la enseñanza de nociones sobre fuerzas en primer ciclo de la escuela primaria. Revista de enseñanza de la física, 26, 265-273.

Pro Bueno, A. (2013). Enseñar procedimientos: por qué y para qué. Alambique, 73, 69-76

Rivero, A., del Pozo, R. M. \& Porlán, R. (2017). Didáctica de las Ciencias Experimentales en educación primaria. Investigación en la Escuela, 93, 76-80. 